\title{
Desempenho em planejamento, flexibilidade e controle inibitório em crianças com e sem TEA: Efeitos dos sintomas comórbidos de atenção e hiperatividade
}

\author{
Bárbara Dias Fabre \\ Patrícia Silva Lúcio
}

\section{RESUMO}

O Transtorno do Espectro Autista (TEA) é um transtorno do neurodesenvolvimento que provoca dificuldades em várias áreas, incluindo as funções executivas (FE), principalmente na presença de comorbidades. $O$ Transtorno de Déficit de Atenção e Hiperatividade (TDAH) é uma das comorbidades mais frequentes do TEA. A presente pesquisa identificou perfis de FE de crianças com e sem TEA e verificou o efeito da presença de comorbidade (TDAH) nesse perfil. A amostra foi composta por 16 crianças com TEA e 32 controles (mesma sala de aula; emparelhadas por idade, sexo e QI não-verbal avaliado pelo Raven). Foram avaliadas as funções de flexibilidade (Wisconsin), planejamento (Torre de Hanoi) e controle inibitório (Go/No-go) e os pais responderam ao SNAP-IV. Conduziram-se análises multi/univariadas (MANOVA e ANOVA) e testes de qui-quadrado. No contraste entre o grupo clínico e controle, não houve diferenças em flexibilidade ou em planejamento. Houve efeito geral no controle inibitório, que se deveu a diferenças na condição No-go (> erro TEA). A análise de comorbidade (2x2; TEA/controle; TDAH presente/ ausente) apontou que os sintomas de TDAH produziram diferenças em flexibilidade e em controle inibitório no contraste entre crianças com TEA+TDAH e controles sem TDAH. Discute-se as implicações dos resultados para a análise de especificidade dos transtornos.

Palavras-chave: funções executivas, Transtorno do Espectro Autista; Transtorno de Déficit de Atenção e Hiperatividade, comorbidade.

\section{ABSTRACT}

Performance in planning, flexibility, and inhibitory control in children with and without ASD: Effects of comorbid attention and hyperactivity symptoms

Autism Spectrum Disorder (ASD) is a neurodevelopmental disorder that causes difficulties in many areas, including executive functions (EF), especially in the presence of comorbidities. Attention Deficit Hyperactivity Disorder (ADHD) is one of the most common comorbidities of ASD. The present study identified EF profiles of children with and without ASD and verified the effect of the presence of comorbidity (ADHD) on this profile. The sample consisted of 16 children with ASD and 32 controls (same classroom; paired by age, gender, and nonverbal IQ evaluated by Raven). Flexibility (Wisconsin), planning (Hanoi Tower), and inhibitory control (Go/No-go) functions were evaluated and parents responded to the SNAP-IV. Multi and univariate analyzes (MANOVA and ANOVA), and chi-square tests were conducted. In the contrast between the clinical and control groups, there were no differences in flexibility or planning. There was a general effect on inhibitory control, which was due to differences in the No-go condition (> ASD error). Comorbidity analysis $(2 \times 2 ; A S D /$ control; ADHD present/absent) showed that ADHD symptoms produced differences in flexibility and in inhibitory control in the contrast between children with ASD+ADHD and controls without ADHD. We discuss the implications of the results for the analysis of specificity of the disorders.

Keywords: executive functions; Autistic Spectrum Disorder; Attention Deficit Hyperactivity Disorder; comorbidity.

\section{Sobre os autores \\ B.D.F. \\ http://orcid.org/0000-0003- 0160-8125 \\ Universidade Estadual de Lon- drina - Londrina, PR barbarafabre@hotmail.com \\ P. S. L. \\ https://orcid.org/0000-0001- 7125-206X \\ Universidade Estadual de Lon- drina - Londrina, PR pslucio@gmail.com}

\section{Direitos Autorais}

Este é um artigo de acesso aberto e pode ser reproduzido livremente, distribuído, transmitido ou modificado, por qualquer pessoa desde que usado sem fins comerciais. 0 trabalho é disponibilizado sob a licença Creative Commons CC-BY-NC.

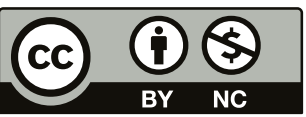




\section{3' INTERACÃO EM PSICOLOGIA}

\section{INTRODUÇÃO}

Segundo a definição da quinta edição do Manual Diagnóstico e Estatísticos de Transtornos Mentais (DSM-V; American Psychiatric Association [APA], 2014), o Transtorno do Espectro Autista (TEA) é um transtorno do neurodesenvolvimento que tem como características a presença de prejuízos nas habilidades interpessoais, falta de contato visual, interesses restritos e comportamentos e movimentos repetitivos. Dessa forma, as crianças com esse transtorno têm dificuldades de socialização, compreensão da comunicação do tipo verbal e não verbal, reciprocidade socioemocional (ou seja, compartiIhar ideias, emoções), dentre outras (APA, 2014).

Não é possível afirmar com exatidão a etiologia do TEA; contudo, há diversas hipóteses sobre influências genéticas e ambientais que sugerem uma heterogeneidade etiológica do transtorno (APA, 2014). Pressupõe-se que alterações presentes nos indivíduos com TEA estejam nas funções executivas (FE) (Antshel et al., 2013; Hill, 2004). Conforme Diamond (2013), as FE, relacionadas às regiões frontais do cérebro, que desempenham papel importante em diversas atividades cotidianas do ser humano, como planejar e estabelecer metas. Assim, déficits de FE podem acarretar prejuízos significativos no funcionamento geral do indivíduo. Pressupõe-se, ainda, que as FE básicas sejam desenvolvidas primeiramente (p.ex., controle inibitório, memória de trabalho e flexibilidade) e dão suporte às funções mais complexas, como o planejamento (Diamond, 2013). Sendo assim, na ocorrência de disfunções executivas, as crianças com TEA podem apresentar dificuldades em ter o controle de suas respostas, planejar e permanecer em determinada tarefa (Hill, 2004).

As dificuldades de FE são maiores quando os sujeitos apresentam quadros de comorbidades (Craig et al., 2016; Lord \& Bischop, 2015). Segundo Alvarenga et al. (2009), o termo "comorbidade" só foi utilizado pela primeira vez no DSM-III-R. Entretanto, conforme Romero et al. (2016), nesta época, o manual ainda não reconhecia a associação de diagnósticos em crianças com TEA, sendo que esta possibilidade ocorreu apenas na sua edição mais recente. Até a quarta edição, os problemas psiquiátricos encontrados em pessoas com autismo eram atribuídos ao transtorno em si. Desde que o DSM-V alterou a caracterização do autismo como uma única categoria dimensional, agrupada em níveis de severidade, os estudos sobre comorbidade no TEA têm sido cada vez mais frequentes na literatura (Romero et al., 2016), possibilitando estabelecer novos padrões e perfis neuropsicológicos do transtorno $(\mathrm{Gr}$ zadzinski et al., 2013).

Moseley et al. (2011) afirmam que o risco de comorbidade no TEA é de duas a quatro vezes maior que em pessoas com desenvolvimento típico. Dados apontam que $70 \%$ de crianças com TEA possuem pelo menos um transtorno comórbido e $40 \%$ com mais de um transtorno (Mansour et al., 2017). 0 transtorno de déficit de atenção e hiperatividade (TDAH) é o segundo maior transtorno comórbido ao TEA, perdendo apenas para os transtornos de ansiedade (Simonoff et al., 2008). Pesquisas têm apresentado diferentes índices de comorbidade entre os transtornos, que em geral dependem das metodologias apresentadas pelos estudos assim como as amostras utilizadas. Antshel et al. (2013) apontam comorbidade de sintomas de TDAH em crianças com TEA entre 31-95\%, enquanto Mansour et al. (2017) indicam que este índice é de 28\%-87\% e Visser et al. (2016) de 17-50\%.

Craig et al. (2016) realizaram uma revisão sistemática de 26 artigos investigando o desempenho das FE de crianças com TDAH, TEA e TEA+TDAH. Os autores encontraram dificuldades comuns entre todos os grupos, a saber, atenção, memória de trabalho, fluência, formação conceitual e processo preparatório. Contudo, crianças com TDAH apresentaram maiores prejuízos no controle inibitório, enquanto as crianças com TEA possuíram dificuldades na flexibilidade e planejamento. Já o desempenho das crianças com TEA + sintomas de TDAH foram mais graves nessas funções avaliadas. Do ponto de vista neuropsicológico, Takeuchi et al. (2013) questionam se o grupo de indivíduos com comorbidade apresentam uma junção simples dos prejuízos de cada transtorno ou se apresentam um padrão de prejuízos específico.

Pelo exposto, as crianças com TEA parecem apresentar prejuízos gerais das FE e, mais especificamente, em flexibilidade e planejamento. Ademais, dificuldades adicionais em controle inibitório aparecem quando apresentam comorbidades com o TDAH (Craig et al., 2016). Apesar de esta ser uma conclusão válida a partir de uma revisão sistemática, observa-se uma grande diversidade metodológica e de amostras dos trabalhos revisados. Por exemplo, o teste de Wisconsin aparece em diferentes estudos na avaliação da flexibilidade, memória de trabalho e inibição de respostas/ formação de conceitos (respectivamente, Ozonoff \& Jensen, 1999; Takeuchi et al., 2013; Kado et al., 2012). Além disso, das 26 revisões realizadas, apenas seis estudos investigaram simultaneamente as funções de flexibilidade, inibição e planejamento, sendo que destes apenas o estudo de Sinzig et al. (2008) avaliou comorbidade TDAH + autismo (estudos citados por Craig et al., 2016). Assim, as tarefas foram selecionadas a partir de um levantamento daquelas que melhor avaliavam os construtos bem como que se adequassem ao presente estudo. Outra característica relevante é de que nenhum estudo brasileiro foi encontrado em termos de investigação de análise de efeito de comorbidade

Nessa direção, esta pesquisa tem dois objetivos centrais: primeiro, investigar se, tal como previsto pela revisão citada, crianças com TEA terão maiores prejuízos em flexibilidade e 


\section{H MTERAC̄OOEM PSICOLOGIA}

planejamento e menor em controle inibitório, comparados ao desempenho de controles sem TEA. Segundo, verificar se a presença de comorbidade com sintomas de TDAH alteram os padrões de desempenho neuropsicológico das crianças com TEA e sem TEA nessas tarefas. As hipóteses deste estudo são de que o grupo TEA (1) tenderá a apresentar dificuldades gerais de FE; mas (2) apenas que as das crianças com sintomas comórbidos de TDAH apresentarão dificuldades em controle inibitório. Para esta finalidade, utilizamos as tarefas disponíveis no Brasil que, pela sua idealização teórica (ver método), são mais apropriadas para a avaliação desses construtos, a saber, a tarefa de Wisconsin para avaliação da flexibilidade, a Torre de Hanoi para a avaliação do planejamento e a tarefa de Go/No-Go para a avaliação do controle inibitório.

\section{MÉTODO}

\section{CONSIDERAÇÕES ÉTICAS}

A presente pesquisa foi iniciada após a aprovação do Comitê de Ética em Pesquisa Envolvendo Seres Humanos (CEP-UEL) sob parecer n. 3.097.119 de 20 de dezembro de 2018 (CAAE: 044.58018.0.0000.5231). Foram avaliadas apenas as crianças cujos pais assinaram o Termo de Consentimento Livre e Esclarecido (TCLE).

\section{CARACTERIZAÇÃO DA PESQUISA E RECRUTAMENTO DA AMOSTRA}

Trata-se de um estudo transversal, do tipo caso-controle, descritivo e comparativo entre grupos. A pesquisa teve como critérios de inclusão estar cursando o ensino regular em escolas municipais da cidade da coleta dos dados e não apresentar queixas de problemas de comportamento. A criança apresentar queixa ou suspeita de deficiência intelectual (DI) e, no caso de ter diagnóstico de TEA, não possuir comunicação verbal foram os critérios de exclusão.

0 recrutamento da amostra se deu a partir da parceria estabelecida com o centro de referência de saúde e de educação de uma cidade do interior do Paraná, que possui o controle de todas as crianças com suspeita ou diagnóstico de transtornos do desenvolvimento ou problemas de aprendizagem no município. Após o contato, a equipe forneceu uma listagem com 33 crianças com diagnóstico de TEA, sem queixa ou suspeita de DI e incluídas no sistema de ensino regular. Destas, 14 foram excluídas por estarem matriculadas em centros de educação infantil (faixa etária fora do escopo da pesquisa). Das 19 restantes, uma mudou de cidade e a outra passou a frequentar a escola especial. Assim, os pais de 17 crianças foram contatados e convidados para participação na pesquisa. Uma dessas crianças foi excluída da amostra, pois não se comunicava verbalmente. Somente após a confirmação do grupo clínico é que se iniciou o agendamento com os responsáveis das crianças do grupo controle. Cada instituição de ensino entrou em contato com os pais dos participantes e agendou uma entrevista, sendo as avaliações realizadas nas próprias escolas para ambos os grupos.

\section{PARTICIPANTES E EMPARELHAMENTO DA AMOSTRA}

Participaram 16 crianças com diagnóstico de TEA (3 meninas), incluídas no sistema regular de escolas municipais, da pré-escola ao Ensino Fundamental, sem suspeita ou queixa de DI. A partir da definição desse grupo, ficou constituído o grupo de controle, isto é, crianças da mesma idade, sexo e sala de aula das crianças com TEA, sorteadas pelas professoras a partir desses critérios. Foi estipulado o quantitativo de dois controles para cada caso. A amostra totalizou 48 participantes (18,8\% meninas). A Tabela 1 apresenta as descritivas da amostra.

Tabela 1. Caracterização da Amostra e Emparelhamento dos Grupos

\begin{tabular}{|c|c|c|c|c|c|c|c|c|}
\hline \multirow{2}{*}{ Grupo } & \multirow{2}{*}{ Ano } & \multicolumn{2}{|c|}{ Idade } & \multicolumn{2}{|c|}{ Sexo } & \multicolumn{2}{|c|}{ Raven } & \multirow[t]{2}{*}{$n$} \\
\hline & & $M$ & DP & $\mathrm{F}$ & $M$ & $M$ & $\mathrm{DP}$ & \\
\hline \multirow{7}{*}{$\begin{array}{l}\frac{0}{0} \\
\stackrel{0}{ \pm} \\
\text { ㅇ } \\
\text { ப }\end{array}$} & Pré & 4,75 & 0,5 & 2 & 2 & 12,50 & 5,50 & 4 \\
\hline & $1^{\circ}$ & 6 & 0,0 & - & 2 & 15,00 & 4,24 & 2 \\
\hline & $2^{\circ}$ & 6,83 & 0,41 & - & 6 & 24,50 & 4,59 & 6 \\
\hline & $3^{\circ}$ & 8,5 & 0,58 & - & 4 & 22,75 & 6,75 & 4 \\
\hline & $4^{\circ}$ & 8,5 & 0,84 & 2 & 4 & 25,66 & 3,07 & 6 \\
\hline & $5^{\circ}$ & 9,8 & 0,79 & 2 & 8 & 27,60 & 5,69 & 10 \\
\hline & Total & 7,67 & 0,54 & 6 & 26 & 23,38 & 7,08 & 32 \\
\hline \multirow{7}{*}{$\underset{\vdash}{\underset{⺊}{\rightleftarrows}}$} & Pré & 4,5 & 0,71 & 1 & 1 & 10,00 & 5,65 & 2 \\
\hline & $1^{\circ}$ & 6 & - & - & 1 & 21,00 & - & 1 \\
\hline & $2^{\circ}$ & 7,67 & 1,16 & - & 3 & 15,66 & 3,51 & 3 \\
\hline & $3^{\circ}$ & 8,5 & 0,71 & - & 2 & 27,50 & 6,36 & 2 \\
\hline & $4^{\circ}$ & 9,33 & 0,58 & 1 & 2 & 24,33 & 9,29 & 3 \\
\hline & $5^{\circ}$ & 10,2 & 0,4 & 1 & 4 & 26,60 & 6,29 & 5 \\
\hline & Total & 8,08 & 0,71 & 3 & 13 & 21,83 & 8,19 & 16 \\
\hline
\end{tabular}

A faixa etária das crianças com TEA não se diferiu do grupo controle $(t(46)=0,693, p=0,492)$. Observou-se que não houve diferenças nos escores entre os grupos TEA e controle em termos de inteligência avaliada pelo Raven $(t(46)=0,668$, $p=0,495)$. Os percentis de inteligência da amostra variaram de 2,3 a 99 e não houve diferenças entre as classificações percentílicas entre os grupos $\left(\chi^{2}(12)=12,771, p=0,386\right)$. 


\section{N"INTERAC̄̃O EM ET PSICOLOGIA}

\section{INSTRUMENTOS E MATERIAIS}

Esta pesquisa faz parte de um estudo maior, que objetiva identificar perfis cognitivos de crianças com e sem TEA, além de verificar o desempenho dessas crianças com a presença de comorbidades. Serão descritos nesta seção apenas os instrumentos e materiais utilizados para os fins desta pesquisa, que buscou identificar o desempenho das crianças nas habilidades de flexibilidade, controle inibitório e planejamento. Nos dois primeiros casos, utilizou-se tarefas computadorizadas a partir de uma adaptação do software gratuito Psytoolkit desenvolvido por Stoet (2010).

Inteligência não verbal (Raven). As Matrizes Progressivas Coloridas de Raven (CPM) - Escala Especial constituem um instrumento utilizado para avaliar raciocínio analógico e habilidade edutiva. É composto por figuras incompletas devendo-se indicar, dentre 6 possibilidades, a parte que a complete. Para cada resposta correta, atribui-se um ponto, sendo o escore máximo 36 pontos (Pasquali et al., 2002).

Flexibilidade (Wisconsin Card Sorting Test - WCST). Para avaliar a flexibilidade, foi utilizada uma versão computadorizada da tarefa original de Berg (1948) que avalia a capacidade simultânea de extrair regras e de modificá-las após sua alteração (Silva-Filho et al., 2011). O baralho é composto por 60 cartas, cada uma contendo de uma a quatro figuras idênticas e da mesma cor, com diferentes tipos de combinação de formas (estrelas, cruzes, triângulos e círculos), cores (vermelho, amarelo, azul e verde) e número. São apresentadas quatro figuras-estímulo e a tarefa do sujeito é categorizar uma carta-alvo de acordo com algum dos critérios (cor, número ou forma). Feedbacks são fornecidos após as respostas. 0 critério muda a cada 10 sequências, com isso, o participante deve descobrir qual o critério a ser utilizado nas próximas tentativas. 0 tempo limite de cada tentativa é de 10 segundos.

Controle inibitório (Go/No-go). A tarefa Go/No-Go foi utilizada para avaliar a capacidade de controle inibitório. $\mathrm{Na}$ versão utilizada, o participante deveria apertar a barra de espaço ao visualizar um círculo verde com a palavra "sim" (Go) no meio e não responder quando aparecia um círculo vermelho com a palavra "não" no meio (No-go). O desempenho na tarefa foi medido pelos erros na versão "sim" (Go) e "não" (No-go) e pelo tempo de resposta nos acertos da condição Go (Criaud, 2013).

Planejamento (Torre de Hanoi). A tarefa consiste em uma plataforma contendo três hastes com três discos sobrepostos de tamanhos diferentes (os menores sob os maiores). A criança deve passar todos os discos para a última torre, na mesma ordem, seguindo regras previamente explicadas (Orsati, 2006). A tarefa é finalizada após acerto ou quando se alcança a quantidade máxima de 15 movimentos. Como medida de desempenho, calcula-se o número de movimentos executados, o tempo para a realização da tarefa e se foi concluída com êxito ou não.

Avaliação de sintomas da atenção e da hiperatividade/impulsividade. O Swanson, Nolan e Pelham Questionnaire (SNAP-IV) é um questionário baseado no DSM-IV, constituído por uma lista de 18 sintomas (9 de desatenção, 3 de impulsividade e 6 de hiperatividade) que devem ser pontuados de 0 a 3 , representando, respectivamente, quatro níveis de gravidade: nem um pouco, um pouco, bastante e demais. Possui estudo de adaptação e validade para a população de crianças brasileiras (Mattos et al., 2006). Para fins desta pesquisa, foi utilizada a versão para pais.

\section{PROCEDIMENTOS}

A partir do contato da escola e agendamento com os pais ou responsáveis, foi realizada uma entrevista individual que incluiu assinatura do TCLE e resposta aos questionários. Essa etapa de coleta de informações durou aproximadamente 60 minutos. A aplicação das tarefas nas crianças também ocorreu de forma individual, entre 40 e 60 minutos. A coleta ocorreu em ambiente fornecido pela própria escola. Os testes padronizados foram aplicados de acordo com informações contidas em seus manuais.

\section{ANÁLISE ESTATÍSTICA}

Conduziram-se análises estatísticas descritivas e inferenciais (escores gerais nas tarefas e por grupos de crianças para os erros e o tempo). Foram apresentados valores de média e desvio-padrão por grupo (e proporções de acertos para variáveis dicotômicas). Nas estatísticas inferenciais de comparação entre os grupos, foram conduzidas análises multivariadas de variância (MANOVA) e análises univariadas de variância (ANOVAS); para variáveis métricas e para comparações entre categorias de respostas, foi utilizado o teste do qui-quadrado $\left(x^{2}\right)$. Utilizou-se a MANOVA nos casos em que as variáveis dependentes (VDs) possuíam diferentes níveis (i.e., categorias de respostas). Nestes casos, o interesse era descobrir como a variável preditora (i.e., pertencer ao grupo de crianças com TEA ou sem TEA) interferiu nos padrões de respostas destes conjuntos de VDs (chamado de média de vetores). Adicionalmente, para saber se os grupos se diferem entre os diferentes níveis, a MANOVA apresenta resultados individuais de ANOVAS. Neste caso, o valor de $p$ foi corrigido para comparações múltiplas (i.e., dividido pelo número de comparações). Foram consideradas significativas as comparações que atingiram valor de $p<0,05$. Para as ANOVAS geradas nas MANOVAS, o valor corrigido de $p$ foi 0,025 para dois níveis e de 0,0125 para quatro níveis. As análises foram conduzidas com o pacote estatístico SPSS versão 23.0. 


\section{H MTERAC̄OOEM ET PSICOLOGIA}

\section{RESULTADOS}

\section{AVALIAÇÃO DO PERFIL COGNITIVO}

A Tabela 2 apresenta as estatísticas descritivas das tarefas aplicadas dentro de cada grupo (valores de média e desvio padrão e frequência de acertos, quando pertinente) e as estatísticas inferenciais correspondentes.

Para a flexibilidade (Wisconsin), não houve diferenças entre os grupos para nenhuma das condições (erro total, erro de perseveração, erro de não perseveração e tempo de reação), seja pelas análises multivariadas ou univariadas. Para o controle inibitório, a análise multivariada mostrou que as diferenças entre os grupos nos compósitos de médias para os erros Go/No-go foram significativas. As análises univariadas mostraram que as diferenças se deveram ao contraste entre os grupos na condição No-go (em função da correção para comparações múltiplas, a condição Go não se mostrou significativa para separar os grupos). Em relação ao tempo nos acertos da condição Go o efeito também não foi significativo.

Na tarefa Torre de Hanoi, apesar de o grupo de controle ter apresentado o dobro da frequência de acertos, as comparações não foram significativas, tampouco para o número de movimentos executados e o tempo de realização da tarefa (Tabela 2). Observações realizadas durante a avaliação mostraram um perfil de ausência de comportamento estratégico pela maioria dos participantes de ambos os grupos, criando um efeito de solo que reduziu a variabilidade. Assim, realizou-se uma análise qualitativa, considerando o desempenho em função do tempo de execução (alto, médio e baixo) determinado pelas diferenças entre a média e um desvio-padrão (para cima e para baixo). Esse procedimento foi adotado pois as crianças que faziam a tarefa muito rápido pareciam violar as regras (p.ex., colocar os três discos juntos no último pino), enquanto as que demoravam muito pareciam mover aleatoriamente os pinos. Para esta análise, considerou-se apenas um controle randomicamente alocado para cada caso (para se evitar efeito de inflação das diferenças por inspeção visual, ser uma análise descritiva).

A Figura 1 apresenta o desempenho em função dessas categorias. Os gráficos não devem ser interpretados como representando variáveis contínuas, mas como linhas ligando os pontos médios de gráficos de barras sobre dois grupos, para melhor visualização das interações. Nenhuma das crianças que realizaram a tarefa em tempos muito alto (comportamento de tentativa e erro) ou muito baixo (desistência ou violação explícita de regras) obteve êxito na tarefa. Entre as que obtiveram tempo médio, a proporção de crianças TEA e controle foi a mesma na condição de erro, mas a prevalência foi duas vezes maior entre as crianças do grupo de controle na condição de acerto.
Tabela 2. Estatísticas Descritivas por Grupo e Resultados das Comparações nas Análises Multivariadas e Univariadas

\begin{tabular}{|c|c|c|c|c|}
\hline \multirow{3}{*}{$\begin{array}{l}\text { Tarefas/ } \\
\text { Variáveis }\end{array}$} & \multicolumn{2}{|c|}{$M(D P)$} & \multirow{2}{*}{ Manova } & \multirow{2}{*}{ Anova ${ }^{1}$} \\
\hline & Controle & TEA & & \\
\hline & \multicolumn{4}{|c|}{ Wisconsin } \\
\hline $\begin{array}{c}P \\
(n=45)\end{array}$ & $\begin{array}{l}16,1 \\
(5,4)\end{array}$ & $\begin{array}{l}15,1 \\
(3,5)\end{array}$ & \multirow{2}{*}{$\begin{array}{c}\lambda(2,42)= \\
1,664, p= \\
202, \eta^{2}= \\
0,073\end{array}$} & $\begin{array}{c}F(1,45)=0,47 \\
p=0,829 \eta^{2}= \\
0,001\end{array}$ \\
\hline $\begin{array}{c}\text { NP } \\
(n=45)\end{array}$ & $\begin{array}{l}11 \\
(5,5)\end{array}$ & $\begin{array}{l}15,1 \\
(8,3)\end{array}$ & & $\begin{array}{c}F(1,45)= \\
3,321, p= \\
0,075, \eta^{2}= \\
0,072\end{array}$ \\
\hline $\begin{array}{c}\text { Erros } \\
(n=45)\end{array}$ & $\begin{array}{l}27,4 \\
(8,8)\end{array}$ & $\begin{array}{l}30,8 \\
(7,2)\end{array}$ & - & $\begin{array}{c}F(1,45)=1,71 \\
p=0,198 \eta^{2}= \\
0,038\end{array}$ \\
\hline \multirow[t]{2}{*}{$\begin{array}{c}\text { TR } \\
(n=42)\end{array}$} & $\begin{array}{c}3098 \\
(1001)\end{array}$ & $\begin{array}{l}3100 \\
(913)\end{array}$ & - & $\begin{array}{c}F(1,42)= \\
0,000, p= \\
0,995, \eta^{2}= \\
0,000\end{array}$ \\
\hline & & Go- & oGo $(n=4 \varepsilon$ & \\
\hline $\begin{array}{l}\text { Go } \\
\text { (E) }\end{array}$ & $\begin{array}{c}0,4 \\
(2,4)\end{array}$ & $\begin{array}{c}3,0 \\
(4,1)\end{array}$ & \multirow{2}{*}{$\begin{array}{c}\lambda(2,45)= \\
4,560, p= \\
0,016, \eta^{2}= \\
0,169\end{array}$} & $\begin{array}{c}F(1,46)= \\
4,901, p= \\
0,032^{1}, \eta^{2}= \\
0,096\end{array}$ \\
\hline $\begin{array}{l}\text { No-go } \\
\text { (E) }\end{array}$ & $\begin{array}{c}0,6 \\
(0,9)\end{array}$ & $\begin{array}{c}1,6 \\
(1,6)\end{array}$ & & $\begin{array}{c}F(1,46)= \\
7,758, p= \\
0,008, \eta^{2}= \\
0,144\end{array}$ \\
\hline \multirow[t]{2}{*}{$\begin{array}{l}\text { Go } \\
\text { (T) }\end{array}$} & $\begin{array}{c}573 \\
(150)\end{array}$ & $\begin{array}{c}668 \\
(160)\end{array}$ & - & $\begin{array}{c}F(1,46)= \\
3,907, p= \\
0,054, \eta^{2}= \\
0,078\end{array}$ \\
\hline & \multicolumn{4}{|c|}{ Torre de Hanoi $(n=48)$} \\
\hline Acertos $^{2}$ & $25 \%$ & $12,5 \%$ & - & $\begin{array}{c}x^{2}(1)=1,011 \\
p=0,315\end{array}$ \\
\hline Movimentos & $\begin{array}{l}10,4 \\
(4,1)\end{array}$ & $\begin{array}{c}8,3 \\
(9,9)\end{array}$ & - & $\begin{array}{c}F(1,46)= \\
2,497, p= \\
0,121, \eta^{2}= \\
0,051\end{array}$ \\
\hline Tempo & $\begin{array}{c}52,1 \\
(36,1)\end{array}$ & $\begin{array}{c}43,8 \\
(38,7)\end{array}$ & - & $\begin{array}{c}F(1,46)= \\
0,536, p= \\
0,468, \eta^{2}= \\
0,012\end{array}$ \\
\hline
\end{tabular}

Nota. NP = erro de não perseveração; $P=$ erro de perseveração; $\mathrm{TR}=$ tempo de reação $(\mathrm{ms}) ; \mathrm{E}=$ Erro; $\mathrm{T}=$ tempo; ${ }^{1}$ Nas anovas univariadas que se seguiram às análises multivariadas das variáveis Wisconsin e Go/No-go, o valor de $p$ foi corrigido pelo número de comparações devido ao efeito de comparações múltiplas (isto é, um novo valor crítico de $p$ foi gerado para que as comparações fossem consideradas significativas). Para as demais variáveis destacadas que possuem comparações múltiplas, o valor de $p$ foi dividido por 2 (i.e., $0,05 / 2=0,025)$. ${ }^{2} 0$ escore é a proporção de crianças que acertaram a tarefa, portanto, a estatística foi o qui-quadrado. Em negrito, comparações significativas. 


\section{NINTERACÃO EM ET. PSICOLOGIA}

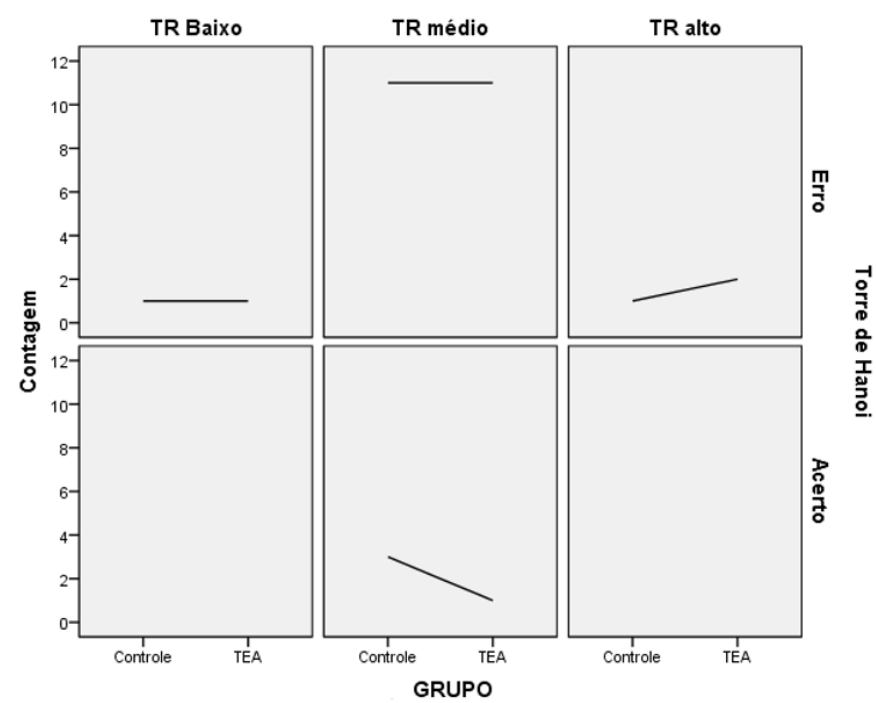

Figura 1. Gráfico de desempenho dos grupos (TEA e controles) na Torre de Hanoi em função do tempo de execução na tarefa.

\section{ANÁLISE DE EFEITO DA COMORBIDADE}

Para investigação dos sintomas de TDAH, foi utilizada a avaliação dos pais por meio do SNAP-IV. No grupo de controle, a proporção de crianças com/ sem TDAH pelo SNAP foi de, respectivamente, $87,5 \%$ e $12,5 \%$, enquanto para o grupo TEA os pais avaliaram $50 \%$ das crianças apresentando alguma classificação de TDAH. A Tabela 3 apresenta os valores de média e desvio padrão dos grupos TEA e controle, com ou sem sintomas de TDAH nas três tarefas.

Para a flexibilidade, a análise multivariada não mostrou efeito geral nos erros de perseveração e não perseveração. De maneira semelhante, as análises univariadas não mostraram diferenças nas condições separadas entre os grupos. Entretanto, as análises de post hoc (com correção de Bonferroni) mostraram que as médias de erros de perseveração das crianças com TEA + TDAH foram maiores do que as crianças do grupo controle sem sintomas de TDAH $(p=0,048)$. Para o controle inibitório, a MANOVA mostrou que os grupos diferiram em termos dos erros (Go e No-go). Entretanto, as ANOVAS univariadas mostraram que o efeito se mostrou significativo apenas para os erros na situação No-go. As análises de post hoc (com correção de Bonferroni) mostraram que as diferenças nesta condição se restringiram às médias de erro das crianças TEA + TDAH e as crianças do grupo controle sem TDAH, com menores médias de erros para este último $(p=0,006)$. Em relação ao tempo, não houve diferenças entre os grupos.

A Figura 2 apresenta o gráfico de desempenho entre os grupos com se sem comorbidade nas tarefas Go/No-Go (acima) e Wisconsin (abaixo). Também neste gráfico, as linhas apenas ligam o ponto médio de um gráfico de barras que incide sobre as categorias de grupos.

Para o planejamento, apesar de as crianças do grupo TEA+TDAH terem apresentado mais erros e terem executado a tarefa em menor tempo e com menos movimentos, as diferenças não atingiram significância em nenhuma das condições. Pelos valores das médias da Tabela 3, nota-se que as crianças que acertam pertencem ao grupo que não possuem sintomas de TDAH, independentemente da condição clínica.

\section{DISCUSSÃo}

Este estudo apresentou dois objetivos centrais, os quais foram: investigar as habilidades de FE de planejamento, flexibilidade e controle inibitório em crianças com TEA, comparando com o desempenho de crianças sem TEA, e verificar o efeito da comorbidade dentro dessa comparação. A hipótese, a partir da revisão realizada por Craig et al. (2016), era de que as crianças com TEA apresentariam maiores problemas em planejamento e flexibilidade e menor em controle inibitório. Os resultados não corroboraram com esta hipótese, pois em nenhuma das habilidades pareceu haver um prejuízo específico relacionado ao TEA. Pelo contrário, as análises quantitativas e qualitativas realizadas sinalizaram para diferenças entre os grupos carregadas pelos sintomas comórbidos ao TDAH.

Durante a coleta de dados, ficou evidente a dificuldade das crianças de ambos os grupos compreenderem o comando da tarefa utilizada para a avaliação do planejamento (torre de Hanoi). Por consequência, houve um efeito de solo (i.e., apenas $25 \%$ das crianças do grupo de controle e $12,5 \%$ do grupo TEA obtiveram êxito). Orsati (2006) avaliou habilidades de FE em grupo de 10 crianças com transtorno invasivo do desenvolvimento e autismo, pareadas com 10 crianças controle. A autora encontrou $40 \%$ de acerto na tarefa de três pinos da Torre de Hanoi e $100 \%$ de acerto entre o grupo de controle. Entretanto, a média de idade das crianças era de 11 anos, bem maior que do presente estudo.

Nossos resultados contrapõem aos achados de Geurts et al. (2004), que investigaram a habilidade de planejamento de crianças TEA, TDAH e controles na tarefa de Torre de Londres. As crianças com TEA apresentaram maior tempo de execução na tarefa do que as crianças com TDAH e os controles, o que corrobora com os achados de Craig et a. (2016) sobre as dificuldades de planejamento serem específicas do autismo. Geurts et al. (2004) não observaram diferenças significativas nos erros ou no tempo de decisão (i.e., tempo entre o término da instrução e o início da atividade). Essa variável não foi investigada no presente estudo. Outra diferença é que os autores utilizaram escores de regressão padronizados para conduzir as MANOVAS e ANOVAS, o que pode ter favorecido o aparecimento de diferenças naquele estudo. 
Tabela 3. Estatísticas Descritivas e de Comparações entre os Grupos Com e Sem Comorbidade

\begin{tabular}{|c|c|c|c|c|c|c|}
\hline Variável & Grupo & TDAH & $\mathrm{M}(\mathrm{DP})$ & Manova & Anova & Post-Hoc \\
\hline & & & & Wiscon & & \\
\hline \multirow{4}{*}{$\mathrm{P}$} & & sem & $16,0(5,6)$ & \multirow{8}{*}{$\begin{array}{c}\lambda(2,40)= \\
1,327, p= \\
0,255, \eta 2= \\
0,093\end{array}$} & & \\
\hline & & com & $16,2(3,5)$ & & $F(3,41)=0,080, p$ & \\
\hline & & sem & $11,8(5,5)$ & & $=0,971 \eta^{2}=0,006$ & NS \\
\hline & ТЕА & com & $15,1(3,0)$ & & & \\
\hline \multirow{4}{*}{ NP } & Controle & sem & $11,1(5,8)$ & & & \\
\hline & & com & $12,2(2,2)$ & & $F(3,41)=2,643, p$ & TEA+TDAH > \\
\hline & & sem & $11,8(5,5)$ & & $=0,062 \eta 2=0,162$ & Controle sem \\
\hline & ТЕА & com & $18,7(9,7)$ & & & \\
\hline \multirow{4}{*}{ Erros } & Controle & sem & $27,9(9,4)$ & \multirow{4}{*}{ - } & & \multirow{4}{*}{ NS } \\
\hline & comtiole & com & $28,5(4,2)$ & & $F(3,43)=0,947 p=$ & \\
\hline & & sem & $28,1(3,5)$ & & $0,426 \eta 2=0,091$ & \\
\hline & IEA & com & $33,8(9,2)$ & & & \\
\hline \multirow{4}{*}{ TR } & & sem & 3261 (1023) & \multirow{4}{*}{-} & & \multirow{4}{*}{ NS } \\
\hline & controle & com & $2613(491)$ & & $F(3,42)=1,399 p=$ & \\
\hline & \multirow{2}{*}{ TEA } & sem & 2754 (838) & & $0,256 \eta 2=0,062$ & \\
\hline & & com & 3561 (859) & & & \\
\hline & & & & Go-No & & \\
\hline \multirow{4}{*}{ Go (E) } & & sem & $1,0(2,5)$ & \multirow{8}{*}{$\begin{array}{c}\lambda(2,43)= \\
2,333, p= \\
0,039, \eta 2= \\
0,140\end{array}$} & & \multirow{4}{*}{ NS } \\
\hline & controle & com & $0,0(0,0)$ & & $F(3,44)=2,207, p$ & \\
\hline & & sem & $2,1(3,4)$ & & $=0,101, \eta^{2}=0,131$ & \\
\hline & IEA & com & $3,8(4,6)$ & & & \\
\hline \multirow{4}{*}{ No-go (E) } & Controle & sem & $0,6(0,8)$ & & & \multirow{4}{*}{$\begin{array}{l}\text { TEA+TDAH > } \\
\text { Controle sem }\end{array}$} \\
\hline & & com & $0,5(1,0)$ & & $F(3,44)=4,377, p$ & \\
\hline & & sem & $1,0(1,0)$ & & $=0,009 \eta^{2}=0,230$ & \\
\hline & IEA & com & $2,2(1,9)$ & & & \\
\hline \multirow{4}{*}{ Go $(T)$} & & sem & 579 (156) & \multirow{4}{*}{ - } & & \multirow{4}{*}{ NS } \\
\hline & controle & com & 535 (118) & & $F(3,44)=1,763, p$ & \\
\hline & \multirow{2}{*}{ TEA } & sem & $626(170)$ & & $=0,168 \eta^{2}=0,107$ & \\
\hline & & com & $710(160)$ & & & \\
\hline & \multicolumn{6}{|c|}{ Torre de Hanoi } \\
\hline \multirow{4}{*}{ Acerto } & \multirow{2}{*}{ Controle } & sem & $28,6 \%$ & \multirow{4}{*}{ - } & & \multirow{4}{*}{ NS } \\
\hline & & com & $0,0 \%$ & & $x^{2}(1)=1,011, p=$ & \\
\hline & & sem & $25,0 \%$ & & 0,315 & \\
\hline & & com & $0,0 \%$ & & & \\
\hline & & sem & $10,4(3,9)$ & & & \\
\hline Mov & controle & com & $10,5(5,4)$ & - & $F(1,44)=1,770, p$ & NS \\
\hline IVIOV. & & sem & $10,1(5,5)$ & - & $=0,167 \eta 2=0,108$ & NS \\
\hline & IEA & com & $6,5(3,7)$ & & & \\
\hline & Controle & sem & $53,5(37,5)$ & & & \\
\hline Tempo & क्षाזा & com & $42,0(24,9)$ & - & $F(1,46)=0,536, p$ & NS \\
\hline rempo & & sem & $57,7(50,2)$ & - & $=0,468 \eta 2=0,012$ & 100 \\
\hline & IEA & com & $29,8(15,5)$ & & & \\
\hline
\end{tabular}

Nota. NP = erro de não perseveração; $\mathrm{P}=$ erro de perseveração; $\mathrm{TR}=$ tempo de reação $(\mathrm{ms}) ; \mathrm{E}=$ Erro; $\mathrm{T}=$ tempo; Mov. = movimentos; $\mathrm{Em}$ negrito, comparações significativas. 


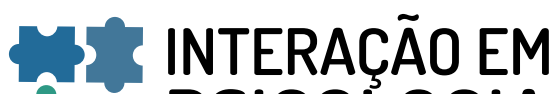 ET PSICOLOGIA}
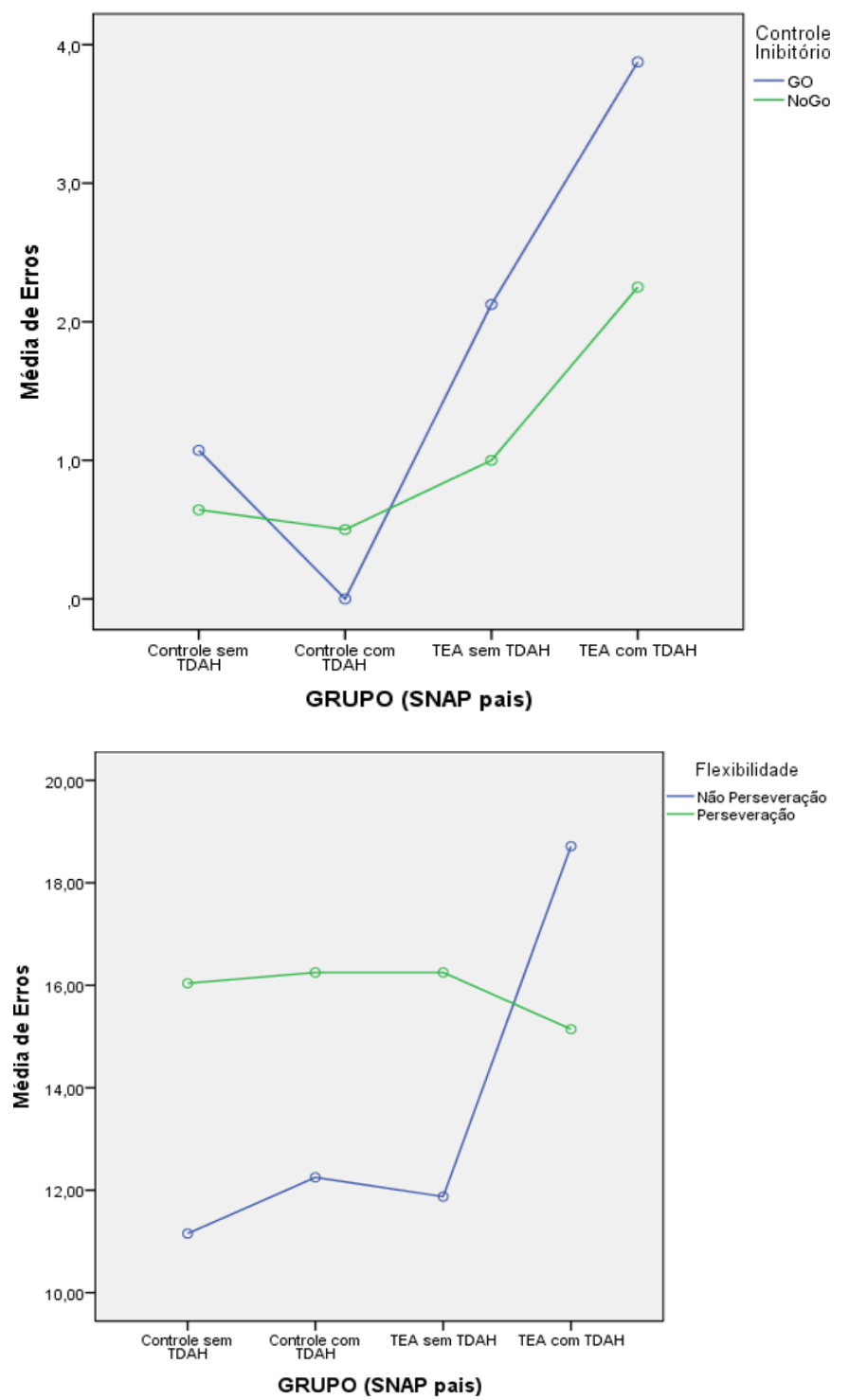

Figura 2. Gráficos de desempenho dos grupos com e sem comorbidade na tarefa Go/No-go (acima) e no Wisconsin (abaixo).

Em relação à flexibilidade, a literatura afırma que crianças com TEA tendem a ter maiores escores de perseveração comparadas ao grupo controle (p. ex., Robinson et al., 2009), ou seja, menor capacidade de adaptação a novas regras. Situações do cotidiano comumente prejudicadas por dificuldades na flexibilidade cognitiva são a rigidez no padrão de comportamento, a presença de dificuldades nas mudanças da rotina ou mesmo dificuldade do reconhecimento facial (Kenworthy et al, 2008; Vries \& Geurts, 2012).

Nossa pesquisa mostrou ausência de diferenças em todas as comparações (erro de perseveração, não perseveração, total de erros e tempo de reação). Resultados semelhantes foram encontrados por Goldberg et al. (2005), que compararam crianças com TEA, TDAH e controles utilizando uma tarefa de set-shifting para avaliar a flexibilidade. Por outro lado, Corbett et al. (2009) encontraram que crianças com TEA, comparadas ao grupo controle e TDAH, apresentavam prejuízos na flexibilidade avaliada pela habilidade de interferência nome/cor. Já Geurst et al. (2004) encontraram pior desempenho de crianças com TEA em relação a controles e crianças com TDAH no Wisconsin. A grande variedade de tarefas utilizadas na literatura dificulta uma comparação direta entre os resultados. No que tange ao Wisconsin, cujas diferenças foram encontradas por Geurst et al. (2004), mas não neste estudo, é possível que a modalidade de apresentação (aqui realizada no computador) e o número de tentativas, que foi mais reduzida que a versão original, tenham interferido nos resultados.

Para o controle inibitório, a MANOVA mostrou diferença significativa entre erro Go e erro No-go, sendo que as diferenças entre os grupos se restringiram à condição de erro No-go (análise univariada). Na revisão realizada por Craig et al. (2016), metade dos estudos encontrou diferenças em controle inibitório em crianças com TEA e TDAH comparados a controles e a outra metade não foi capaz de identificar diferenças. Desse modo, é ainda controverso na literatura se o controle inibitório, de fato, está alterado em crianças com TEA em relação às crianças típicas. Geurst et al. (2004) apontaram déficits de controle inibitório em crianças com TEA comparados a controles, utilizando uma tarefa de Stop Signal, mas este grupo de crianças não se diferiu das crianças com TDAH.

As análises de comorbidade permitiram verificar a presença de perfis neuropsicológicos nas tarefas. Para o planejamento, a proporção de acertos nos grupos de crianças sem sintomas de TDAH foi aproximada, respectivamente, $25 \%$ e $28,5 \%$ para as crianças com TEA e controle (Tabela 3). De maneira análoga, independentemente do grupo, as crianças que apresentaram sintomas de TDAH não apresentaram acertos. Isso implica que, mesmo com a ausência de diferença significativas, talvez possa existir um padrão de respostas, ou seja, um pior desempenho quando os sintomas de TDAH estão presentes. Para a flexibilidade, a análise post hoc mostrou que as diferenças se restringiram às comparações entre o grupo TEA com TDAH e o controle sem sintomas, na condição de não perseveração. Isso pode indicar que as crianças com TEA + TDAH tiveram dificuldades em aprender a tarefa, uma vez que os erros de não perseveração indicam como a criança identifica as regras (o que pode ter feito com que respondessem por tentativa e erro). Finalmente, para o controle inibitório, a comparação significativa ocorreu entre os grupos TEA + TDAH e os controles sem TDAH, na condição No-go (que é a indicadora da inibição da resposta na tarefa). 


\section{W'INTERAC̄̃O EM ET PSICOLOGIA}

A partir do exposto, em todas as funções comparadas, a presença de sintomas de TDAH agravou o prejuízo das habilidades das crianças do grupo clínico. Os dados podem indicar, ao contrário da revisão de Craig et al. (2016), que os sintomas da atenção e hiperatividade permeiam, de um modo geral, as dificuldades de crianças nas tarefas executivas consideradas, não havendo subsídios, a partir de nossos dados, para distinguir uma especificidade do TEA nas tarefas de planejamento ou flexibilidade.

Os resultados obtidos podem sofrer alterações com o aumento do tamanho da amostra ou com a inclusão de crianças com diagnóstico de TDAH nas comparações. De fato, o único estudo revisado por Craig et al. (2016) que mais diretamente se relaciona a esta pesquisa (i.e., Sinzig et al., 2008) também encontrou resultados divergentes com a conclusão geral da revisão, pois apenas o planejamento pareceu haver especificidade para as crianças com autismo, sendo as diferenças em flexibilidade restritas ao grupo com comorbidade. 0 uso de análise univariada de variância neste estudo pode ter forçado o aparecimento do efeito, mas optamos aqui pela MANOVA para reduzir possibilidade de erro Tipo I em função de comparações múltiplas.

Sinzig et al. (2008) também utilizaram a proporção de 1 caso para 1 controle, o que também pode inflar as diferenças entre grupos. Esses argumentos são corroborados pelos tamanhos de efeito encontrados ( $d$ de Cohen), que foram em sua maioria pequenos (calculados a partir das médias de desvios apresentados) e pelas diferenças terem aparecido apenas em sub-habilidades das FE avaliadas (p. ex., número de erros ou tempo). Estudos futuros devem focar em metodologias mais unitárias, estatísticas mais robustas e em definições operacionais das tarefas para a seleção das habilidades investigadas a fim de iluminar as complexas relações entre os sintomas psicopatológicos e as alterações do neurodesenvolvimento.

\section{CONSIDERAÇÕES FINAIS}

Esta pesquisa comparou o desempenho de crianças com TEA e controles em um conjunto de habilidades de FE que, conforme Craig et al. (2016), poderiam estar relacionados a déficits específicos a este grupo de crianças, distinguindo-as daquelas que apresentam TDAH. Os resultados questionam a validade dos achados que indicam que o TEA está relacionado especificamente a déficits em planejamento e flexibilidade, enquanto o TDAH está associado a dificuldades no controle inibitório. A análise de comorbidade mostrou que todas estas dificuldades executivas estão especificamente, de uma forma ou outra, vinculadas aos sintomas de TDAH.
Obviamente, esta conclusão não é definitiva. Nossa amostra, de caráter clínico, apresentou poucas crianças, o que há implicações para sua heterogeneidade e limitações do poder estatístico. Ademais, a classificação das crianças conforme a opinião dos pais no SNAP não indica que as crianças, de fato, apresentavam comorbidade com o TDAH. Estudos considerando crianças com diagnóstico comórbidos devem ser realizados, a exemplo da literatura (p.ex., Antshel et al., 2013; Boxhoorn et al., 2018; Tye, 2013).

Apesar dessas limitações, ressalta-se que o estudo é relevante para futuras investigações que busquem compreender as frequentes comorbidades entre os transtornos TEA e TDAH ao apontar para a necessidade de um esforço por parte dos pesquisadores em melhor definir as FE e formas de avaliação. Ademais, possibilita ampliar o olhar para o desenvolvimento de intervenções que envolvam a dimensão das disfunções executivas das crianças, o que permite contribuir para o aprimoramento do funcionamento e qualidade de vida desta população (Simonoff et al., 2008).

\section{AGRADECIMENTOS}

As autoras agradecem ao Centro de Apoio Multiprofissional Escolar (CAME) da cidade de Apucarana - PR pela parceria e coleta de dados e a todas as escolas, pais e crianças envolvidas.

\section{DECLARAÇÃO DA CONTRIBUIÇÃO DOS AUTORES}

A pesquisa faz parte da dissertação de mestrado da primeira autora, orientada pela segunda. Certificamos que as autoras participaram suficientemente do trabalho para tornar pública sua responsabilidade pelo conteúdo. A contribuição de cada autor pode ser atribuída como se segue:

B.D.F. realizou a idealização do projeto que deu base ao artigo, coletou e planilhou os dados, contribuiu para a conceitualização e pela revisão final do artigo. P.S.L. orientou a elaboração do trabalho, a idealização do artigo, realizou a análise dos dados e participou da revisão final.

\section{DECLARAÇÃO DE CONFLITOS DE INTERESSE}

Os autores declaram que não há conflitos de interesse no manuscrito submetido.

\section{DECLARAÇÃO DE FINANCIAMENTO}

A pesquisa relatada no manuscrito foi financiada pelos próprios autores. 


\section{W MTERAC̄̄oEM PSICOLOGIA}

\section{REFERÊNCIAS}

American Psychiatric Association. (2014). DSM-5: Manual diagnóstico e estatístico de transtornos mentais. Artmed Editora.

Antshel, K. M., Zhang-James, Y., \& Faraone, S. V. (2013). The comorbidity of ADHD and autism spectrum disorder. Expert Review of Neurotherapeutics, 13(10), 1117-1128. https://doi.org/10.1586/14737175.2013.840417

Alvarenga, M. A. S., Flores-Mendoza, C. E., \& Gontijo, D. F. (2009). Evolução do DSM quanto ao critério categorial de diagnóstico para o distúrbio da personalidade antissocial. Jornal Brasileiro de Psiquiatria, 58(4), 258-266. https://doi.org/10.1590/S0047-20852009000400007

Berg, E. A. (1948). A simple objective technique for measuring flexibility in thinking. The Journal of general psychology, 39(1), 15-22. https://doi.org/10.1080/00221309.1948.9918159

Boxhoorn, S., Lopez, E., Schmidt, C., Schulze, D., Hänig, S., \& Freitag, C. M. (2018). Attention profiles in autism spectrum disorder and subtypes of attention-deficit/hyperactivity disorder. European Child \& Adolescent Psychiatry, 27(11) 1433-1447. https://doi.org/10.1007/s00787-018-1138-8.

Corbett, B. A., Constantine, L. J., Hendren, R., Rocke, D., \& Ozonoff, S. (2009). Examining executive functioning in children with autism spectrum disorder, attention deficit hyperactivity disorder and typical development. Psychiatry Research, 166(2-3), 210-222. https://doi.org/10.1016/j.psychres.2008.02.005

Criaud, M., \& Boulinguez, P. (2013). Have we been asking the right questions when assessing response inhibition in go/ no-go tasks with fMRI? A meta-analysis and critical review. Neuroscience \& biobehavioral reviews, 37(1), 11-23. https://doi.org/10.1016/j.neubiorev.2012.11.003

Craig, F., Margari, F., Legrottaglie, A. R., Palumbi, R., De Giambattista, C., \& Margari, L. (2016). A review of executive function deficits in autism spectrum disorder and attention-deficit/ hyperactivity disorder. Neuropsychiatric Disease And Treatment, 12, 1191-1202 https://doi.org/10.2147/NDT.S104620

Diamond, A. (2013). Executive functions. Annual Review of Psychology, 64, 135-168. https://doi.org/10.1146/annurev-psych-113011-143750

Geurts, H. M., Verté, S., Oosterlaan, J., Roeyers, H., \& Sergeant, J. A. (2004). How specific are executive functioning deficits in attention deficit hyperactivity disorder and autism? Journal of Child Psychology and Psychiatry, 45(4), 836-854. https://doi.org/10.1111/j.1469-7610.2004.00276.x

Goldberg, M. C., Mostofsky, S. H., Cutting, L. E., Mahone, E. M., Astor, B. C., Denckla, M. B., \& Landa, R. J. (2005). Subtle executive impairment in children with autism and children with ADHD. Journal of Autism and Developmental Disorders, 35(3), 279-293. https://doi.org/10.1007/s10803-005-3291-4
Grzadzinski, R., Huerta, M., \& Lord, C. (2013). DSM-5 and autism spectrum disorders (ASDs): An opportunity for identifying ASD subtypes. Molecular Autism, 4(1), 12-12. https:// doi.org/10.1186/2040-2392-4-12

Hill, E. L. (2004). Executive dysfunction in autism. Trends in Cognitive Sciences, 8(1), 26-32. https://doi.org/10.1016/j. tics.2003.11.003

Kado, Y., Sanada, S., Yanagihara, M., Ogino, T., Ohno, S., Watanabe, K., Ohtsuka, Y. (2012). Executive function in children with pervasive developmental disorder and attention-deficit/hyperactivity disorder assessed by the Keio version of the Wisconsin card sorting test. Brain and Development, 34(5), 354-359. https://doi.org/10.1016/j.braindev.2011.08.008

Kenworthy, L., Yerys, B. E., Anthony, L. G., \& Wallace, G. L. (2008). Understanding executive control in autism spectrum disorders in the lab and in the real world. Neuropsychology Review, 18(4), 320-338. https://doi.org/10.1007/ s11065-008-9077-7

Lord, C., \& Bishop, S. L. (2015). Recent advances in autism research as reflected in DSM- 5 criteria for autism spectrum disorder. Annual Review of Clinical Psychology, 11, 53-70. https://doi.org/10.1146/annurev-clinpsy-032814-112745

Mansour, R., Dovi, A. T., Lane, D. M., Loveland, K. A., \& Pearson, D. A. (2017). ADHD severity as it relates to comorbid psychiatric symptomatology in children with Autism Spectrum Disorders (ASD). Research in Developmental Disabilities, 60, 52-64. https://doi.org/10.1016/j.ridd.2016.11.009

Mattos, P., Pinheiro, M. A., Rohde, L. A. P., \& Pinto, D. (2006). Apresentação de uma versão em português para uso no Brasil do instrumento MTA-SNAP-IV de avaliação de sintomas de transtorno do déficit de atenção/hiperatividade e sintomas de transtorno desafiador e de oposição. Revista de Psiquiatria do Rio Grande do Sul, 28, 290-297. https:// doi.org/10.1590/S0101-81082006000300008

Moseley, D. S., Tonge, B. J., Brereton, A. V., \& Einfeld, S. L. (2011). Psychiatric comorbidity in adolescents and young adults with autism. Journal of Mental Health Research in Intellectual Disabilities, 4(4), 229-243. https://doi.org/10.1 080/19315864.2011.595535

Orsati, F. (2006). Autismo e transtornos invasivos do desenvolvimento: Avaliação neuropsicológica. Cadernos de Pós-Graduação em Distúrbios do Desenvolvimento, 6(1). http:// editorarevistas.mackenzie.br/index.php/cpgdd/article/ view/11148

Ozonoff, S., \& Jensen, J. (1999). Brief report: Specific executive function profiles in three neurodevelopmental disorders. Journal of Autism and Developmental Disorders, 29(2), 171-177. https://doi.org/10.1023/A:1023052913110

Pasquali, L., Wechsler, S., \& Bensusan, E. (2002). Matrizes Progressivas do Raven Infantil: um estudo de validação para o Brasil. Avaliação Psicológica, 1(2), 95-110. 


\section{- IN INERACÃO EM LFICOLOGIA}

Robinson, S., Goddard, L., Dritschel, B., Wisley, M., \& Howlin, P. (2009). Executive functions in children with Autism Spectrum Disorders. Brain and Cognition, 71(3), 362-368. https://doi.org/10.1016/j.bandc.2009.06.007

Romero, M., Aguilar, J. M., Del-Rey-Mejías, Á., Mayoral, F., Rapado, M., Pecina, M., Barbrancho, M. A., Ruiz-Verguila, M. R., \& Lara, J. P. (2016). Psychiatric comorbidities in autism spectrum disorder: A comparative study between DSM-IV-TR and DSM-5 diagnosis. International Journal of Clinical and Health Psychology, 16(3), 266-275. https://doi.org/10.1016/j.ijchp.2016.03.001

Silva-Filho, J. H. D., Pasian, S. R., \& Humberto, J. S. M. (2011). Teste Wisconsin de classificação de cartas: Uma revisão sistemática de 1952 a 2009. Psico-USF, 16(1), 107-116.

Simonoff, E., Pickles, A., Charman, T., Chandler, S., Loucas, T., \& Baird, G. (2008). Psychiatric disorders in children with autism spectrum disorders: Prevalence, comorbidity, and associated factors in a population-derived sample. Journal of the American Academy of Child \& Adolescent Psychiatry, 47(8), 921-929. https://doi.org/10.1097/ CHI.0b013e318179964f

Sinzig, J., Morsch, D., Bruning, N., Schmidt, M. H., \& Lehmkuhl, G. (2008). Inhibition, flexibility, working memory and planning in autism spectrum disorders with and without comorbid ADHD-symptoms. Child and Adolescent Psychiatry and Mental health, 2(4). https://doi.org/10.1186/1753-2000-2-4
Stoet, G. (2010). PsyToolkit: A software package for programming psychological experiments using Linux. Behavior Research Methods, 42(4), 1096-1104.

Takeuchi, A., Ogino, T., Hanafusa, K., Morooka, T., Oka, M., Yorifuji, T., \& Ohtsuka, Y. (2013). Inhibitory function and working memory in attention deficit/hyperactivity disorder and pervasive developmental disorders: Does a continuous cognitive gradient explain ADHD and PDD traits?. Acta Medica Okayama, 67(5), 293-303. https://doi.org/10.18926/ amo/51865

Tye, C., Asherson, P., Ashwood, K. L., Azadi, B., Bolton, P., \& McLoughlin, G. (2013). Attention and inhibition in children with ASD, ADHD and co-morbid ASD+ ADHD: An event-related potential study. Psychological Medicine, 44(5), 11011116. https://doi.org/10.1017/S0033291713001049

Visser, J. C., Rommelse, N. N., Greven, C. U., \& Buitelaar, J. K. (2016). Autism spectrum disorder and attention-deficit/ hyperactivity disorder in early childhood: A review of unique and shared characteristics and developmental antecedents. Neuroscience \& Biobehavioral Reviews, 65, 229263. https://doi.org/10.1016/j.neubiorev.2016.03.019

Vries, M., \& Geurts, H. M. (2012). Cognitive flexibility in ASD: Task switching with emotional faces. Journal of Autism and Developmental Disorders, 42(12), 2558-2568. https:// doi.org/10.1007/s10803-012-1512-1 